\title{
Zoledronic acid impairs oral cancer stem cells by reducing CCL3
}

\author{
SUNG HEE LEE ${ }^{1}$, NICOLE RIGAS ${ }^{1}$, CHARLOTTE ELLEN MARTIN ${ }^{1}$, ANTHONY NGUYEN ${ }^{1}$, \\ MO K. KANG ${ }^{1,2}$, NO-HEE PARK ${ }^{1-3}$, REUBEN H. KIM ${ }^{1,2}$ and KI-HYUK SHIN ${ }^{1,2}$ \\ ${ }^{1}$ The Shapiro Family Laboratory of Viral Oncology and Aging Research, UCLA School of Dentistry; \\ ${ }^{2}$ UCLA Jonsson Comprehensive Cancer Center; ${ }^{3}$ Department of Medicine, \\ David Geffen School of Medicine at UCLA, Los Angeles, CA 90095, USA
}

Received June 26, 2019; Accepted December 19, 2019

DOI: $10.3892 /$ or.2020.7835

\begin{abstract}
Cancer stem-like cells (CSCs; also referred to as tumor-initiating cells) play crucial roles in tumor progression and aggressiveness. Recent studies have demonstrated the antitumor activity of zoledronic acid (ZA), a third-generation bisphosphonate, in various types of human cancer. However, its effect on oral CSCs and the underlying mechanism remain obscure. The present study demonstrated that ZA suppresses the growth and stemness properties of oral/oropharyngeal squamous cell carcinoma (OSCC) cells. ZA inhibited the malignant characteristics of OSCC cells, such as anchorage-independent growth and epithelial thickening in organotypic raft cultures. Moreover, ZA treatment resulted in suppression of self-renewal capacity, a key feature of CSCs. ZA also inhibited important CSC properties, such as migration and chemo-radioresistance. Mechanistically, ZA exposure significantly decreased chemokine (C-C motif) ligand 3 (CCL3) expression in OSCC cells. It was further demonstrated that CCL3 signaling via its receptor is crucial for supporting the CSC phenotype in OSCC cells. Moreover, an antagonist of the CCL3 receptor reversed the effect of CCL3 on CSC properties, and exogenous CCL3 rescued the suppressaed CSC phenotype in ZA-treated OSCC cells. These results demonstrated that ZA suppresses the CSC phenotype in OSCC cells by reducing CCL3 expression, suggesting that ZA may be an effective therapeutic agent for oral cancer by targeting CSCs.
\end{abstract}

\section{Introduction}

Oral/oropharyngeal squamous cell carcinoma (OSCC) is a common malignant tumor of the head and neck, and its overall 5 -year relative survival rate is $\sim 50 \%$, urgently requiring new

Correspondence to: Dr Ki-Hyuk Shin or Dr Reuben H. Kim, UCLA School of Dentistry, Center for The Health Sciences, Room 43-003, 10833 Le Conte Avenue, Los Angeles, CA 90095, USA

E-mail:kshin@dentistry.ucla.edu

E-mail: rkim@dentistry.ucla.edu

Key words: zoledronic acid, oral/oropharyngeal squamous cell carcinoma, cancer stem cells, chemokine (C-C motif) ligand 3 directions in therapeutics (1). Cancer stem-like cells (CSCs; also referred to as tumor-initiating cells) are small subpopulations of tumor cells that have been isolated from various primary tumors and cancer cell lines, including OSCC (2). CSCs play a crucial role in tumorigenicity, metastasis and recurrence and, thus, are considered as the origin of the cancer (2). The stemness properties of CSCs are supported by endogenous CSC signaling pathways, such as Notch, Hedgehog, Wnt, Bmil, phosphatase and tensin homolog, bone morphogenetic protein and transforming growth factor- $\beta$, which are frequently activated in human cancers (3). In addition, novel oral CSC molecular determinants, such as histone demethylases (4), microRNA (5), human papilloma viruses (5) and calcium signalling (6) were recently identified. Therefore, advancing our understanding of the molecular properties and signaling pathways unique to oral CSCs is crucial for developing a new generation of targeted and effective therapies for OSCC.

Nitrogen-containing bisphosphonates (N-BPs) are commonly used anti-resorptive agents in the treatment of bone-related diseases, such as osteoporosis and metastatic bone tumors. Zoledronic acid (ZA) is the most potent N-BP available clinically, and its antitumor effects have been demonstrated in a number of solid tumors, including OSCC $(7,8)$. ZA can inhibit cancer cell growth by inducing apoptosis, cell cycle arrest, and by stimulating autophagy (7,9-12), which are crucial for tumor growth and survival. Moreover, the antitumor effect of ZA was also demonstrated in various cancer mouse models, such as breast (13) and pancreas (14), indicating that ZA is an effective agent for controlling human cancer. Indeed, clinical trials of ZA as an adjuvant therapy have been conducted for breast cancer $(15,16)$. However, the effects of ZA on oral CSCs and its underlying mechanism of action have not been fully elucidated.

The aim of the present study was to demonstrate the inhibitory effects of ZA on the CSC phenotype in OSCC cells, in order to determine whether chemokine (C-C motif) ligand 3 (CCL3) signaling may be of value as a novel downstream target of ZA-induced CSC suppression.

\section{Materials and methods}

Cell culture and reagents. Six human OSCC cell lines, namely SCC4, UM17B, UM5, UM2, BapT and SCC9/TNF (17), 
were cultured in DMEM/Ham's F12 (Invitrogen; Thermo Fisher Scientific, Inc.) supplemented with 10\% FBS (Gemini Bioproducts) and $0.4 \mu \mathrm{g} / \mathrm{ml}$ hydrocortisone (Sigma-Aldrich; Merck KGaA). ZA (LKT Laboratories), human CCL3 (Cell Signaling Technology, Inc.), etoposide (Sigma-Aldrich; Merck $\mathrm{KGaA}$ ), methotrexate (Sigma-Aldrich; Merck KGaA) and Maraviroc (Sigma-Aldrich; Merck KGaA) were purchased and used in the present study.

Anchorage-independent growth. Cells $(10,000)$ were seeded in $60-\mathrm{mm}$ dishes in culture medium containing $0.3 \%$ agarose over a base layer of serum-free medium containing $0.5 \%$ agarose, as described in our prior studies (4-6,17). After 3 weeks of incubation with the indicated ZA concentrations, the cell colonies were counted. The experiment was performed in triplicates.

Organotypic raft cultures. The detailed protocol of this assay was described in our previous publications $(5,17)$. The mean epithelial thickness was obtained from 10 randomly selected epithelial layers formed by OSCC cells. The thickness was measured by Aperio's ImageScope v.12.4 (Aperio Technologies, Inc.).

Tumorsphere formation assay. Cells $(3,000)$ were grown in $3 \mathrm{ml}$ of tumorsphere medium in Ultra-Low Attachment 6-well Plates (Corning, Inc.) for 6-10 days $(4-6,17)$. The medium contained serum-free DMEM/F12 supplemented with 1:50 B27 (Invitrogen; Thermo Fisher Scientific, Inc.), $20 \mathrm{ng} / \mathrm{ml}$ EGF, $20 \mathrm{ng} / \mathrm{ml}, 10 \mu \mathrm{g} / \mathrm{ml}$ insulin, penicillin, streptomycin and amphotericin B.

Side population analysis. Cells $(1,000,000)$ were incubated in culture medium containing Hoechst $33342(5 \mu \mathrm{g} / \mathrm{ml}$; Thermo Fisher Scientific, Inc.) for $90 \mathrm{~min}$ at $37^{\circ} \mathrm{C}$ with or without (1 or $2.5 \mu \mathrm{M}$ ZA. The cells were then spun down, resuspended in ice-cold PBS containing 7-aminoactinomycin D ( $2 \mu \mathrm{g} / \mathrm{ml}$; Thermo Fisher Scientific, Inc.), and subjected to fluorescence-activated cell sorting analysis. The Hoechst dye was excited with the UV laser at 351 to $364 \mathrm{~nm}$ and its fluorescence measured with a $515-\mathrm{nm}$ side population filter (Hoechst blue) and a 608 EFLP optical filter (Hoechst red). The assay was performed and analyzed by the UCLA Flow Cytometry Core.

Migration assay. Cell migration was measured using $6.5-\mathrm{mm}$ Transwell chambers with $8.0-\mu \mathrm{m}$ polycarbonate membranes (cat. no. 3422; Corning, Inc.) as described in our previous publications $(4-6,17)$. Cells $(20,000)$ were seeded with or without $1 \mu \mathrm{M} \mathrm{ZA}$ and incubated for 2 days.

Chemo-radiosensitivity assays. Chemosensitivity was determined by measuring cell viability using the tetrazolium salt (MTT) cell proliferation assay kit (American Type Culture Collection). The detailed protocol is described in our previous studies $(4,17)$. The cells were plated at a density of $2 \times 10^{3}$ cells per well into 96-well plates and incubated in culture medium containing $40 \mu \mathrm{M}$ etoposide or $40 \mu \mathrm{M}$ methotrexate, with or without ZA, for 4 days. Absorbance at $570 \mathrm{~nm}$ was determined using a microplate reader (Synergy H1; BioTek Instruments,
Inc.). For the radiosensitivity assay, the cells were irradiated with 5 Gy using Mark I-30 Cesium-137 irradiator (JL Shepherd \& Assoc.) with a delivery rate of $4.86 \mathrm{~Gy} / \mathrm{min}$, and cultured in growth medium containing 0 or $1 \mu \mathrm{M} \mathrm{ZA}$ for 10 days. Subsequently, surviving colonies were stained and counted. The detailed protocol was as previously described (17).

Western blotting. Western blotting was performed as previously described (4-6,17). The following primary antibodies were used: Notch intracellular domain (NICD; cat. no. 2421; 1:500; Val1744; Cell Signaling Technology, Inc.), $\beta$-catenin (1:500; cat. no. 9562; Cell Signaling Technology, Inc.), and GAPDH (1:1,000; cat. no. sc-25778; Santa Cruz Biotechnology, Inc.). The secondary horseradish peroxidase-conjugated antibody (cat. no. 7074; Cell Signaling Technology, Inc.) dilution range was $1: 2,000$ or $1: 4,000$.

Reverse transcription-quantitative PCR (RT-qPCR). cDNA was synthesized from $2.5 \mu \mathrm{g}$ total RNA using the SuperScript First-Strand Synthesis system (Invitrogen; Thermo Fisher Scientific, Inc.). Subsequently, qPCR was performed using SYBR Green I Master mix (Roche Diagnostics) and LightCycler 480 II (Roche Diagnostics) as described in our previous studies $(4-6,17)$. All primer sequences were obtained from the Universal Probe Library (Roche Diagnostics), and may be made available upon request. The second derivative $\mathrm{Cq}$ value determination method (Roche Diagnostics $\mathrm{GmbH}$ ) was used to compare fold-differences according to the manufacturer's instructions.

Statistical analysis. The statistical analyses were performed using GraphPad Prism 5 (GraphPad Software, Inc.). The data are expressed as mean \pm standard deviation. Data among multiple groups were compared using one-way ANOVA with Newman-Keuls test, while data between two groups were compared using t-tests. $\mathrm{P}<0.05$ was considered to indicate statistically significant differences.

\section{Results}

ZA suppresses the malignant growth properties of OSCC cells. To investigate the effect of ZA on OSCC growth, 6 OSCC cell lines were exposed to increasing concentrations of ZA $(0-20 \mu \mathrm{M})$ for 4 days, and their growth was determined using the MTT assay. ZA decreased the growth of 6 OSCC cell lines in a dose-dependent manner; however, there was a significant difference in the response to ZA among the tested OSCC cell lines (Fig. S1). To examine the effect of ZA on malignant growth properties, its effect on anchorage-independent growth ability of OSCC cells was investigated. Soft agar assay revealed that OSCC cells exposed to ZA exhibited a significant reduction in colony formation at minimal cytotoxic doses of ZA (1-5 $\mu \mathrm{M}$; Fig. 1A). A 3D organotypic cell culture system was also employed, whereby squamous epithelium was reconstituted $(17,18)$. As shown in Fig. 1B, OSCC cells exposed to ZA exhibited significantly reduced epithelial thickness compared with the unexposed control cells. Indeed, previous studies have demonstrated successful inhibition of tumor growth by ZA in vivo $(9,11)$. These findings collectively suggest that ZA suppresses malignant growth of OSCC cells. 
A

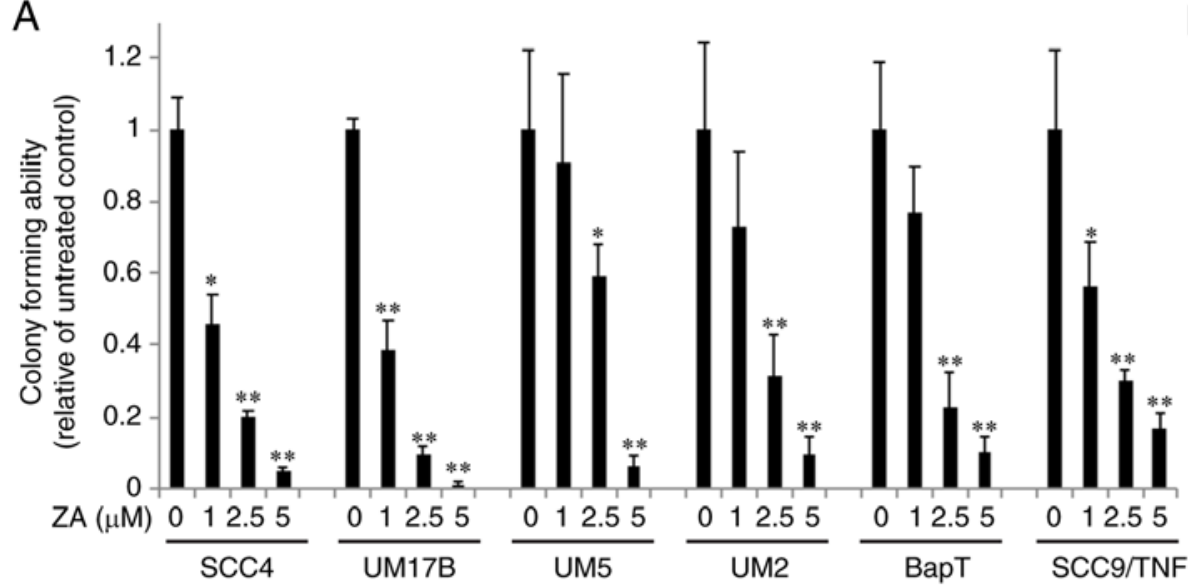

B

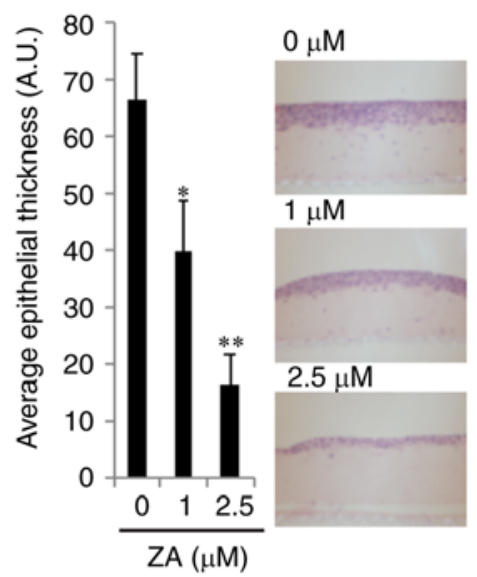

C

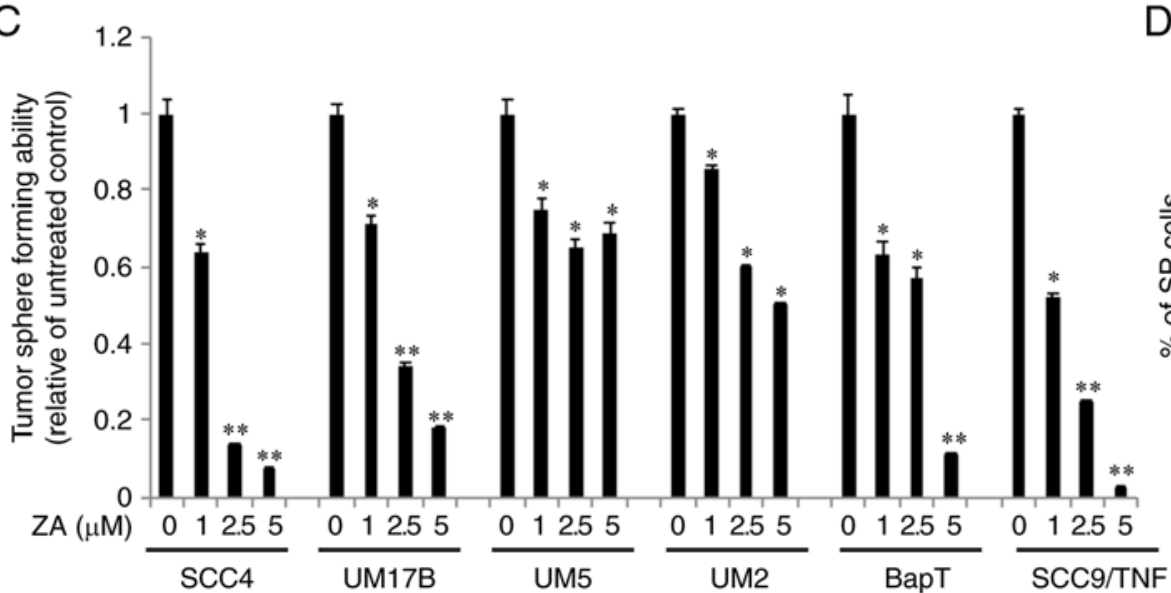

D

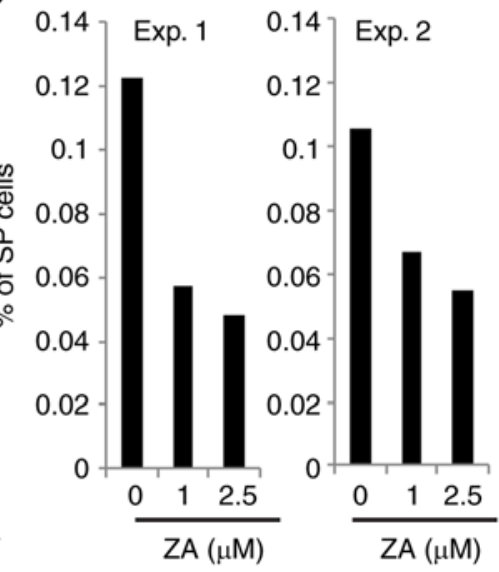

Figure 1. ZA suppresses malignant growth properties and the CSC population in OSCC. (A) The effect of ZA on anchorage-independent growth ability of 6 OSCC cell lines was determined by soft agar assay. Data are presented as means \pm standard deviation of triplicate experiments. (B) The effect of ZA on epithelial thickening was determined by ex vivo three-dimensional organotypic raft cultures. Organotypic raft cultures were established with UM17B cells exposed to different ZA concentrations $(0,1$ and $2.5 \mu \mathrm{M})$, and their mean epithelial thickness was obtained from 10 randomly selected epithelial layers. A representative image of the squamous epithelium formed is shown on the right (magnification, $\mathrm{x} 40$ ). (C) Effect of ZA on the self-renewal capacity of 6 OSCC cell lines was determined by tumorsphere formation assay. ${ }^{*} \mathrm{P}<0.05$ and ${ }^{* *} \mathrm{P}<0.01$ vs. the untreated control in one-way ANOVA. (D) Effect of ZA on SP cells in SCC4 cells was determined by Hoechst 33342 FACS analysis. The assay was independently repeated twice (Exp.1 and Exp. 2). ZA, zoledronic acid; CSC, cancer stem-like cells; OSCC, oral/oropharyngeal squamous cell carcinoma; SP, side population.

ZA suppresses the CSC phenotype. Self-renewal capacity is a key feature of CSCs and is considered to be pivotal for tumorigenic potential (2). Thus, the effect of ZA on the self-renewal capacity of OSCC cells was investigated by performing tumorsphere formation assay. OSCC cells exposed to minimal doses of ZA demonstrated a significant reduction in tumorsphere formation (Fig. 1C), indicating that ZA suppresses the self-renewal capacity of OSCC cells. The effect of ZA on side population (SP) cells, which are considered as a stem-like cell population among a heterogeneous OSCC cell population, was further investigated $(19,20)$. ZA diminished the number of SP cells (Fig. 1D). We further examined the effect of ZA on other CSC properties, i.e., migration and sensitivity to chemoradiotherapy (2). As demonstrated by a Transwell migration assay (Fig. 2A), ZA strongly inhibited the motility in OSCC cells. ZA sensitized OSCC cells to radiation (Fig. 2B) and to chemotherapeutic drugs, i.e., etoposide and methotrexate (Fig. 2C). As expected, 1.0 $\mu \mathrm{M}$ ZA alone did not inhibit OSCC cell growth. ZA slightly potentiated the cytotoxic effect of chemotherapeutic drugs and radiation on OSCC cells. Taken together, these findings indicate that ZA suppresses the CSC phenotype in OSCC cells.

The CSC phenotype can be maintained by several endogenous signaling pathways, including Notch and Wnt (2). Thus, we sought to test whether ZA suppresses the CSC phenotype through targeting these signaling pathways. The effect of ZA on Notch signaling was examined by measuring the expression of proteolytic release of NICD, indicative of Notch activation, in the control and ZA-treated cells. The expression of NICD was not altered by ZA in SCC4 and UM17B cells (Fig. 2D). The effect of ZA on Wnt signaling was also determined by measuring the expression of $\beta$-catenin, the key transcription factor in Wnt signaling. ZA exerted no effect on the expression of $\beta$-catenin (Fig. 2D) and its downstream targets (data not shown). Taken together, these results suggest the involvement of other CSC-related signaling pathways in the ZA-mediated CSC inhibition.

$Z A$ reduces the CCL3 expression required to support the CSC phenotype in OSCC cells. The role of cytokines in the 
A

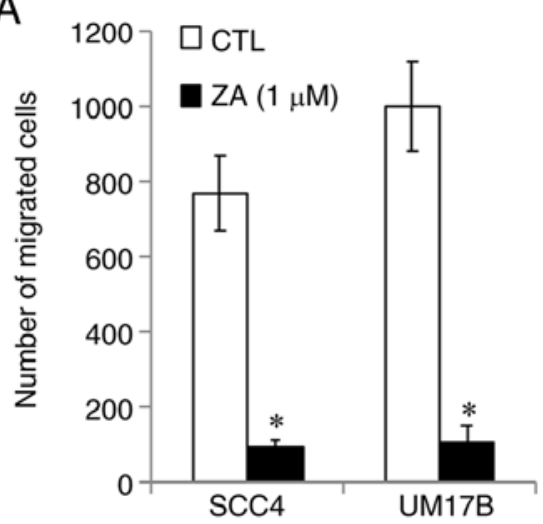

SCC4/CTL

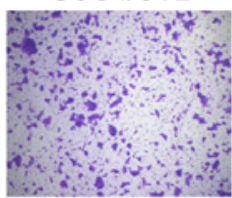

SCC4/ZA

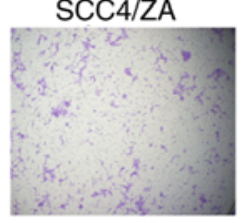

UM17B/CTL

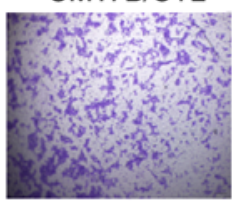

UM17B/ZA

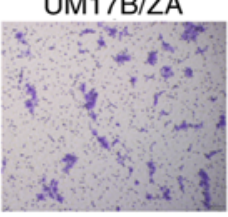

B

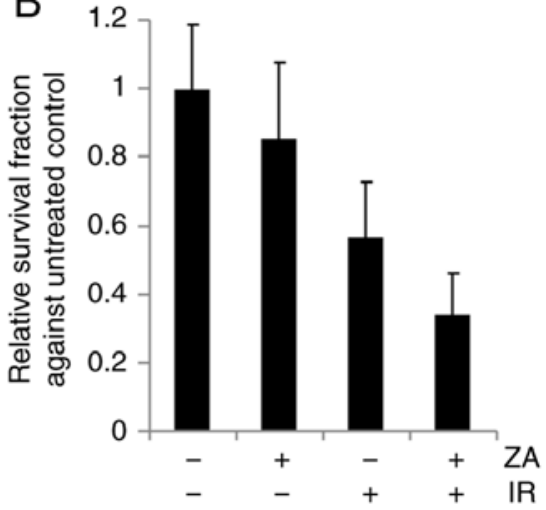

C
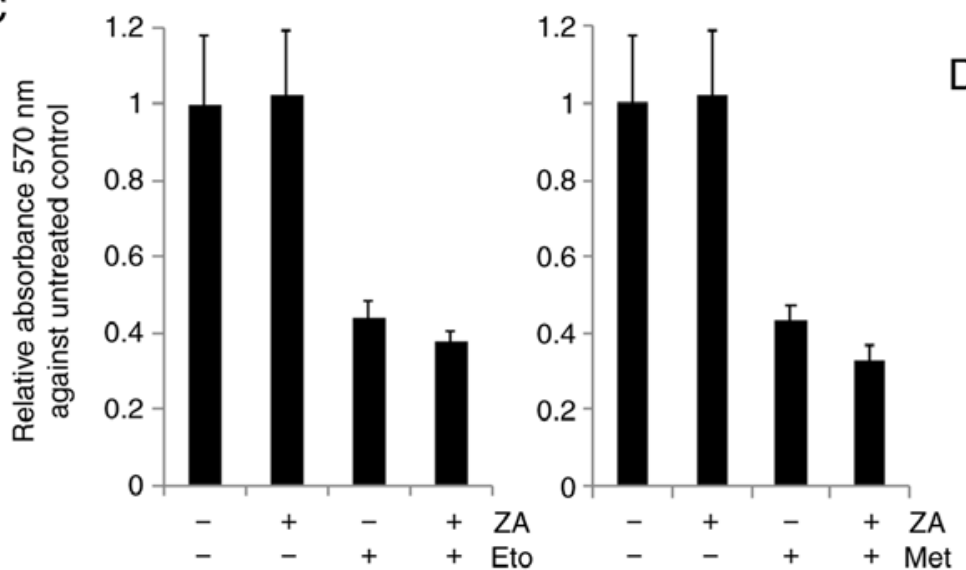

D

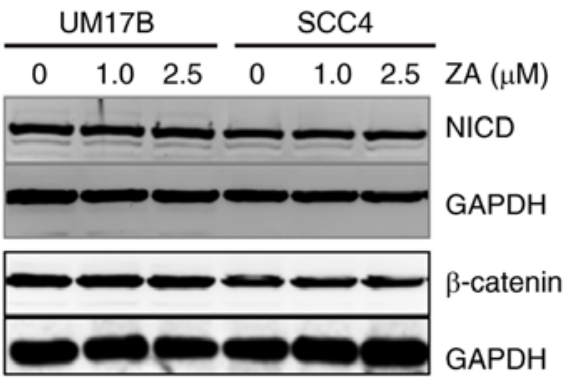

Figure 2. ZA inhibits CSC properties in OSCC cells. (A) The effect of ZA on the migration ability of OSCC cell lines (SCC4 and UM17B) was determined using Transwell chambers. Migration ability was described as the number of migrated cells per field, with data presented as mean \pm standard deviation for 3 randomly selected fields. " $\mathrm{P}<0.01$ vs. the untreated control (CTL) by t-test. Representative images of the migration assay are shown on the right. (B) The effect of ZA on the radiosensitivity of SCC4 cells was determined by clonogenic survival assay. The cells were irradiated (IR) with 5 Gy and cultured in the growth medium containing 0 (-) or $1 \mu \mathrm{M} \mathrm{ZA} \mathrm{(+)} \mathrm{for} 10$ days, and the surviving colonies were stained and counted. (C) The effect of ZA on the chemosensitivity of SCC4 cells was determined by MTT assay. The cells were incubated in culture medium containing $40 \mu \mathrm{M}$ etoposide (Eto) or $40 \mu \mathrm{M}$ of methotrexate (Met), with or without ZA, for 4 days. Relative cell survival was expressed and normalized to untreated control. (D) The effect of ZA on CSC signaling pathways (Notch and Wnt) was determined by western blot analysis using NICD and $\beta$-catenin antibody. GAPDH antibody was used as loading control. Cells were treated with the indicated concentrations of ZA for 2 days and harvested for the assay. ZA, zoledronic acid; CSC, cancer stem-like cells; OSCC, oral/oropharyngeal squamous cell carcinoma; NICD, Notch intracellular domain.

CSC phenotype has been demonstrated in various cancers, including OSCC $(4,17)$. Therefore, to determine whether there is a possible functional association between cytokines and the ZA-mediated inhibition of the CSC phenotype, we first profiled the expression of 19 cytokines in control and ZA-treated SCC4 cells (Fig. S2). qPCR analysis demonstrated that IL7, IL8, IL36RN, CCL3, CCL5 and VEGF were markedly reduced by ZA (Figs. S2 and 3A). Among those, the inhibitory effect of ZA on the expression of IL36RN and CCL3 was further confirmed in other OSCC cell lines (Fig. 3A). The present study focused on CCL3, as its role in CSC was demonstrated in human cancer (21).

The expression level of CCL3 was first compared between CSC-enriched and corresponding non-CSC populations. CCL3 was found to be highly enriched in self-renewing CSC populations, i.e., tumorspheres compared with their corresponding non-CSC populations, i.e., adherent monolayer cells derived from SCC4 cells (Fig. 3B). The activity of aldehyde dehydrogenase 1 (ALDH1) has been used as a CSC marker for human cancers, including OSCC (2). ALDH $1^{\text {High }}$ cancer cells display enhanced CSC properties compared with ALDH $1^{\text {Low }}$ cells (2). We observed that sorted ALDH1 ${ }^{\text {High }}$ SCC4 cells expressed higher CCL3 mRNA levels compared with sorted ALDH1 $1^{\text {Low }}$ SCC4 cells (Fig. 3B). The CSC population can be enriched after treatment with chemotherapeutic drugs (2). Thus, the CCL3 expression was also measured in cisplatin-resistant SCC4 cells that were isolated from SCC4 cells treated with $25 \mu \mathrm{M}$ cisplatin for 2 days. CCL3 mRNA was highly expressed in cisplatin-resistant SCC4 cells compared with their parental control SCC4 cells (Fig. 3B). Moreover, the functional role of CCL3 in CSC properties was investigated. Tumorsphere formation assay revealed that CCL3 promoted self-renewal capacity in multiple OSCC cell lines (Fig. 3C). CCL3 also significantly increased the migration of OSCC cells (Fig. 3D). Collectively, these findings indicate that CCL3 is an important regulator of CSCs in OSCC.

To further confirm the importance of CCL3-mediated signaling for the CSC phenotype, the role of CCL3 receptor, CCR5, in the CCL3-mediated CSC regulation was examined. CCR5 binds to CCL3 with a high affinity (22). We examined the effect of the CCR5 antagonist maraviroc on the CCL3-induced CSC phenotype, and found that maraviroc attenuated the promoting effect of CCL3 on CSC properties, 
A $\quad \mathrm{scC} 4$

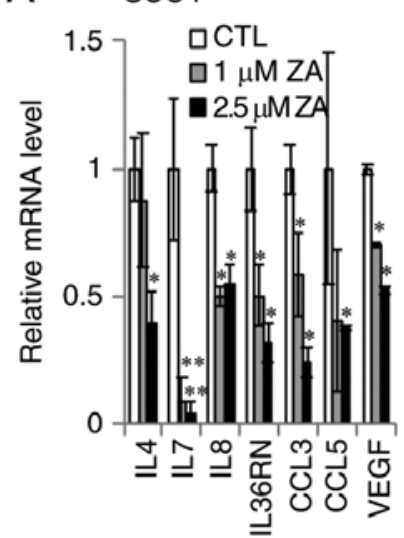

UM17B

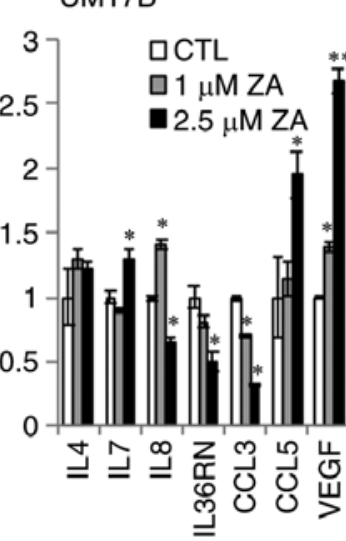

BapT

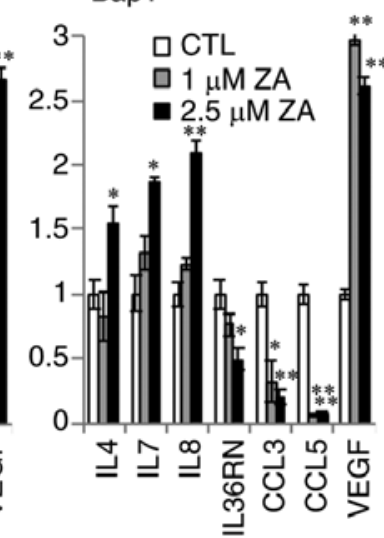

B

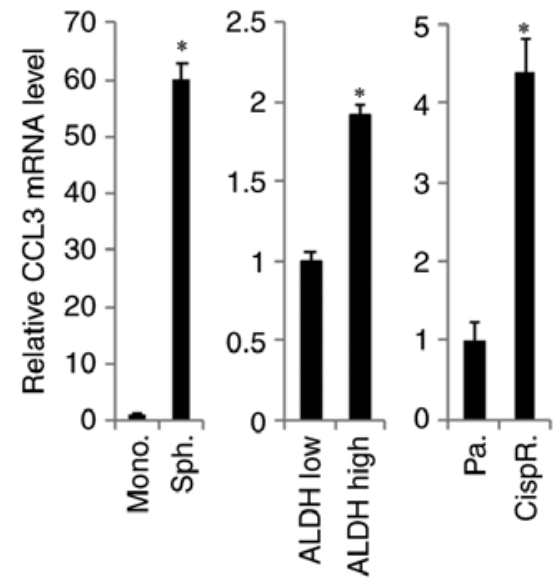

C
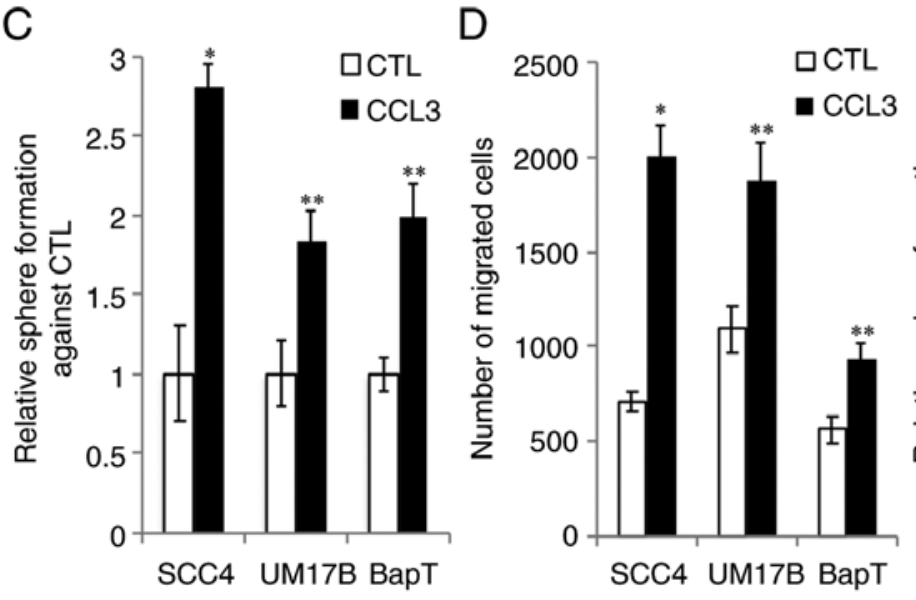

E
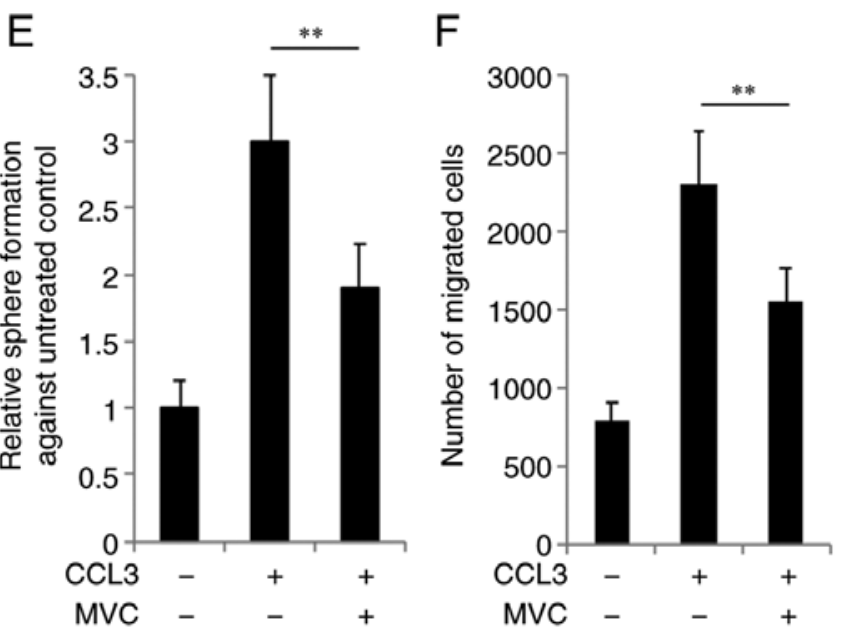

Figure 3. ZA reduces CCL3 expression required to support the CSC phenotype in OSCC. (A) The effect of ZA on CCL3 expression in OSCC was determined by quantitative PCR. Decreased expression of CCL3 was confirmed in 3 OSCC cell lines (SCC4, UM17B and BapT). The cells were treated with the indicated ZA concentrations for 2 days. ${ }^{*} \mathrm{P}<0.05$ and $^{* * *} \mathrm{P}<0.01$ vs. control (CTL) in one-way ANOVA. (B) The level of CCL3 expression was determined in CSC-enriched population and non-CSC population derived from SCC4 cells. Monolayer adherent cells (Mono., non-CSC population) vs. spheres (Sph., CSC-enriched population); $\mathrm{ALDH}^{\text {low }}$ (non-CSC population) vs. $\mathrm{ALDH}^{\text {high }}$ (CSC-enriched population); parental cells (Pa., non-CSC population) vs. cisplatin-resistant cells (CispR., CSC-enriched population). ${ }^{*} \mathrm{P}<0.01$ vs. corresponding non-CSC populations by t-test. (C) The effect of CCL3 on self-renewal capacity was assessed in multiple OSCC cell lines by tumorsphere formation assay. Tumorsphere formation assay was performed without CCL3 (CTL) or with $10 \mathrm{ng} / \mathrm{ml} \mathrm{CCL3}$ (CCL3). ${ }^{*} \mathrm{P}<0.01$ and ${ }^{* *} \mathrm{P}<0.05$ compared to CTL by t-test. (D) The effect of CCL3 on the migration ability of OSCC cells was determined by Transwell assay. ${ }^{*} \mathrm{P}<0.01$ and ${ }^{* *} \mathrm{P}<0.05$ compared to CTL by t-test. (E) The effect of maraviroc (MVC) on CCL3-induced self-renewal capacity of SCC4 cells was assessed by tumorsphere formation assay. The assay was performed in medium containing no treatment (-), $10 \mathrm{ng} / \mathrm{ml} \mathrm{CCL3} \mathrm{(+),} \mathrm{or} 100 \mu \mathrm{M}$ MVC (+) for 7 days. ${ }^{* *} \mathrm{P}<0.05$ in one-way ANOVA. (F) The effect of MVC on CCL3-induced migration in SCC4 cells was assessed by Transwell assay. The assay was performed with no treatment (-), $10 \mathrm{ng} / \mathrm{ml} \mathrm{CCL3} \mathrm{(+),} \mathrm{or} 100 \mu \mathrm{M}$ MVC (+) for $48 \mathrm{~h} .{ }^{* *} \mathrm{P}<0.05$ in one-way ANOVA. ZA, zoledronic acid; CSC, cancer stem-like cells; OSCC, oral/oropharyngeal squamous cell carcinoma; CCL3, chemokine (C-C motif) ligand 3; ALDH, aldehyde dehydrogenase; IL, interleukin; VEGF, vascular endothelial growth factor.

such as self-renewal capacity (Fig. 3E) and migration ability of OSCC cells (Fig. 3F). Maraviroc at $100 \mu \mathrm{M}$ was used, as this concentration exerts no cytotoxic effects on OSCC cells for 7 days (data not shown). Taken together, these findings indicate that the CCL3/CCR5 axis plays an important role in regulating CSCs, further suggesting a functional involvement of CCL3 in ZA-mediated CSC inhibition.

Exogenous CCL3 rescues CSC properties in ZA-treated OSCC cells. We hypothesized that ZA suppresses the CSC phenotype via reduction of CCL3 expression. To test this hypothesis, we investigated whether CCL3 can rescue the CSC properties suppressed by ZA. Tumorsphere formation assays revealed that addition of recombinant human CCL3 rescued self-renewal capacity in the ZA-treated OSCC cells
(Fig. 4A). Similarly, CCL3 also rescued migration ability in the ZA-treated OSCC cells (Fig. 4B). These findings indicate that addition of recombinant human CCL3 can rescue the CSC properties suppressed by ZA. Finally, we examined whether CCL3 upregulates CSC-promoting factors, and observed no significant changes in their expression by CCL3 (Fig. 4C). Collectively, these data indicate that decreased CCL3 expression is likely a major cause of the ZA-mediated inhibition of the CSC phenotype, suggesting a novel CSC inhibitory mechanism by ZA.

\section{Discussion}

The present study demonstrated the antitumor effects of ZA on OSCC. ZA inhibited the malignant growth properties of 

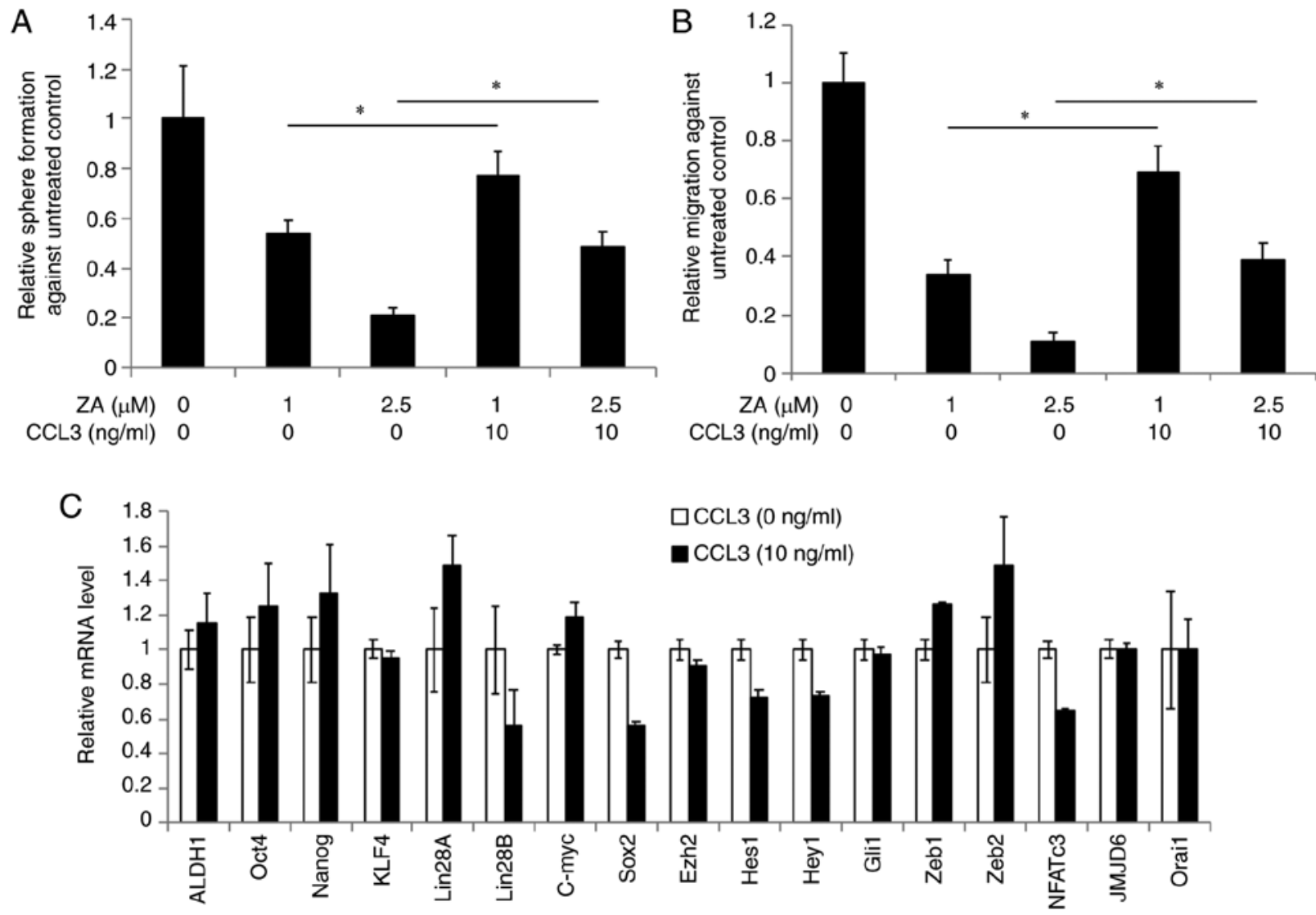

Figure 4. CCL3 rescues CSC properties in ZA-treated OSCC cells. (A) The rescue effect of CCL3 on self-renewal capacity was determined in SCC4 cells treated with 0,1 or $2.5 \mu \mathrm{M}$ ZA by tumorsphere formation assay. CCL3 was added at 0 or $10 \mathrm{ng} / \mathrm{ml}$ in the sphere medium. ${ }^{*} \mathrm{P}<0.05$ in one-way ANOVA. (B) The rescue effect of CCL3 on migration ability was determined in the ZA-treated SCC4 cells by Transwell assay. "P<0.05 in one-way ANOVA. (C) The effect of CCL3 on CSC-related gene expression was evaluated by quantitative (q)PCR. SCC4 cells were treated with CCL3 for $24 \mathrm{~h}$ and subjected to qPCR analysis. ZA, zoledronic acid; CSC, cancer stem-like cells; OSCC, oral/oropharyngeal squamous cell carcinoma; CCL3, chemokine (C-C motif) ligand 3.

OSCC cells, such as anchorage-independent growth and squamous epithelium formation in organotypic raft cultures. Moreover, ZA treatment led to suppression of self-renewal capacity, which is considered as a key feature of CSCs. ZA also inhibited important CSC properties, including migration and chemo-radioresistance. Interestingly, CCL3, a regulator of the CSC phenotype, was inhibited by ZA and successfully rescued the suppressed CSC properties in ZA-treated OSCC cells. Therefore, ZA may be an effective therapeutic agent for oral cancer via suppressing cancer cell stemness.

CSCs have been identified in a broad spectrum of solid tumors, including OSCC. Similar to normal stem cells, CSCs retain their self-renewal capacity, and are thus responsible for tumorigenicity $(2,3)$. They are also considered as key contributors to manifestations of tumor aggressiveness, such as metastasis and drug resistance. Therefore, targeting CSCs appears to be an effective therapeutic intervention. The present study suggested that ZA inhibits tumor aggressiveness by suppressing CSCs. ZA suppressed not only the number of CSCs, but also important CSC properties. Treatment of OSCC cells with ZA resulted in decreased self-renewing CSCs and SP cells. SP cells comprise a stem-like cell population and have been identified in different types of cancer, including OSCC $(19,20)$. SP cancer cells display a high self-renewal potential $(19,20)$. It was clearly demonstrated that ZA suppressed self-renewal capacity in multiple OSCC cell lines. Moreover, ZA also inhibited important CSC properties, such as migration and chemo-radioresistance. The finding of the present study are consistent with previous reports showing the inhibitory effect of ZA on the migration ability of breast cancer cells (23). In the present study, ZA reversed epithelial-to-mesenchymal transition (EMT), the key cellular process in metastasis, by inactivating nuclear factor- $\mathrm{\kappa B}$ (23). Furthermore, a recent study demonstrated that $\mathrm{ZA}$ reduced the motility of CSCs isolated from breast cancer cells (24). Thus, the effect of ZA on EMT and EMT-related gene expression in OSCC should be further investigated. It was also reported that ZA sensitized OSCC cells to cisplatin and radiation. This observation is consistent with previous reports $(25,26)$. Since CSCs play a key role in tumor growth and aggressiveness, the findings of the present study are of paramount importance for the development of more effective oral cancer therapies by using ZA. However, the underlying mechanism through which ZA suppresses OSCC CSC phenotype has not been fully elucidated.

CCL3 has been shown to play an important role in carcinogenesis. Elevated CCL3 expression was found not only in OSCC cells (27), but also in a carcinogen-induced animal model of oral carcinogenesis (28). CCL3 overexpression has also been associated with poor survival rates of OSCC patients (27). CCL3 can promote cancer cell growth and migration (28-30), whereas inhibition of CCL3 suppressed tumor 
growth and angiogenesis (30) and increased cellular sensitivity to therapeutic drugs (31), suggesting the important role of CCL3 in CSC properties. Indeed, CCL3 was found to be highly expressed in CSC-enriched OSCC cell populations compared with non-CSC populations, and promoted CSC properties, including self-renewal capacity, migration and chemoresistance. Interestingly, our study demonstrated that ZA treatment resulted in decreased CCL3 expression in OSCC cells, suggesting that ZA inhibits OSCC cell CSC phenotype by reducing CCL3. It is well known that CCL3 exerts its biological effects by activating downstream signaling cascades after binding to its receptors, i.e., CCR5 (32). Ectopic CCR5 expression can increase CSC population and phenotype in breast cancer (33). Conversely, inhibition of CCR 5 by its antagonist, maraviroc, reduced tumor growth (34) and CSC properties (35), such as metastasis and chemoresistance. Indeed, the CCL3/CCR5 axis has demonstrated pro-tumorigenic activity in oral carcinogenesis (28). Similarly, it was observed that the CCR5 antagonist attenuated CCL3-induced self-renewal capacity and migration of OSCC cells, suggesting a novel role of the CCL3/CCR5 axis in the regulation of CSC properties. However, the CSC-promoting downstream signaling pathway activated by the CCL3/CCR5 axis remains elusive. Interestingly, a recent study demonstrated that CCL3 treatment resulted in activation of the mitogen-activated protein kinase (MAPK) signaling pathway in cancer cells (30). The MAPK signaling pathway, including extracellular signal-regulated kinase (ERK), plays an important role in malignant tumor growth. Maehara et al demonstrated that an activated ERK signaling pathway is crucial for the maintenance of the CSC numbers and properties in esophageal squamous cell carcinoma (36). Thus, future studies should investigate whether ZA suppresses the CSC phenotype by inhibiting the CCL3/ERK signaling axis. Of note, ZA also inhibited IL36RN expression in multiple OSCC cell lines (Fig. 3A). Since IL36RN expression on cancer cells and its role in CSCs remain unknown, further investigation is required to elucidate the role of IL36RN in OSCC.

In summary, the present study demonstrated the inhibitory effect of ZA on the CSC phenotype in OSCC cells. Identification of CCL3 signaling as a novel downstream target of ZA-induced CSC suppression provides new insight into the mechanism through which ZA exerts its antitumor effects. Since CSCs are considered as key to tumor aggressiveness, targeting CSCs by ZA may be an effective therapeutic approach to the treatment of oral cancer.

\section{Acknowledgements}

The authors would like to thank all members of The Shapiro Family Laboratory of Viral Oncology and Aging Research for discussing data interpretation and sharing their knowledge. Part of this study was presented at the annual meeting of the International Journal of Dental Research, June 2019 and was published as Abstract no. 3394.

\section{Funding}

The present study was supported in part by the UCLA School of Dentistry faculty seed grant and the NIH/NIDCR grant (no. R01DE023348).

\section{Availability of data and materials}

The datasets generated and analyzed in the present study are included in this published article. Data not shown in the manuscript are available from the corresponding author upon reasonable request.

\section{Authors' contributions}

KS and RHK contributed to the study conception, design and manuscript preparation. SHL and NR performed the cell line experiments and biochemical assays. CEM and AN contributed to the acquisition of gene expression data. MKK and NP contributed to data evaluation and writing of the manuscript. All authors have read and approved the final manuscript.

\section{Ethics approval and consent to participate}

The experimental protocols were registered and approved by the UCLA Institutional Biosafety committee (BUA-2016-302-001).

\section{Patient consent for publication}

Not applicable.

\section{Competing interests}

All authors declare that they have no competing interest.

\section{References}

1. Warnakulasuriya S: Global epidemiology of oral and oropharyngeal cancer. Oral Oncol 45: 309-316, 2009.

2. Shin KH and Kim RH: An updated review of oral cancer stem cells and their stemness regulation. Crit Rev Oncog 23: 189-200, 2018.

3. O'Brien CA, Kreso A and Jamieson CH: Cancer stem cells and self-renewal. Clin Cancer Res 16: 3113-3120, 2010.

4. Lee CR, Lee SH, Rigas NK, Kim RH, Kang MK, Park NH and Shin KH: Elevated expression of JMJD6 is associated with oral carcinogenesis and maintains cancer stemness properties. Carcinogenesis 37: 119-128, 2016.

5. Lee SH, Lee CR, Rigas NK, Kim RH, Kang MK, Park NH and Shin KH: Human papillomavirus 16 (HPV16) enhances tumor growth and cancer stemness of HPV-negative oral/oropharyngeal squamous cell carcinoma cells via miR-181 regulation. Papillomavirus Res 1: 116-125, 2015.

6. Lee SH, Rigas NK, Lee CR, Bang A, Srikanth S, Gwack Y, Kang MK, Kim RH, Park NH and Shin KH: Orail promotes tumor progression by enhancing cancer stemness via NFAT signaling in oral/oropharyngeal squamous cell carcinoma. Oncotarget 7: 43239-43255, 2016.

7. Tamura T, Shomori K, Nakabayashi M, Fujii N, Ryoke K and Ito $\mathrm{H}$ : Zoledronic acid, a third-generation bisphosphonate, inhibits cellular growth and induces apoptosis in oral carcinoma cell lines. Oncol Rep 25: 1139-1143, 2011.

8. Martin CK, Werbeck JL, Thudi NK, Lanigan LG, Wolfe TD, Toribio RE and Rosol TJ: Zoledronic acid reduces bone loss and tumor growth in an orthotopic xenograft model of osteolytic oral squamous cell carcinoma. Cancer Res 70: 8607-8616, 2010.

9. Okamoto S, Kawamura K,LiQ, Yamanaka M, Yang S, Fukamachi T, Tada Y, Tatsumi K, Shimada H, Hiroshima K, et al: Zoledronic acid produces antitumor effects on mesothelioma through apoptosis and S-phase arrest in p53-independent and Ras prenylation-independent manners. J Thorac Oncol 7: 873-882, 2012.

10. Corey E, Brown LG, Quinn JE, Poot M, Roudier MP, Higano CS and Vessella RL: Zoledronic acid exhibits inhibitory effects on osteoblastic and osteolytic metastases of prostate cancer. Clin Cancer Res 9: 295-306, 2003. 
11. Jiang $\mathrm{P}$, Zhang $\mathrm{P}$, Mukthavaram R, Nomura N, Pingle SC, Teng D, Chien S, Guo F and Kesari S: Anti-cancer effects of nitrogen-containing bisphosphonates on human cancer cells. Oncotarget 7: 57932-57942, 2016.

12. Sewing L, Steinberg F, Schmidt H and Göke R: The bisphosphonate zoledronic acid inhibits the growth of HCT-116 colon carcinoma cells and induces tumor cell apoptosis. Apoptosis 13 : 782-789, 2008

13. Li Y, Du Y, Sun T, Xue H, Jin Z and Tian J: PD-1 blockade in combination with zoledronic acid to enhance the antitumor efficacy in the breast cancer mouse model. BMC Cancer 18: 669, 2018.

14. Vitellius C, Fizanne L, Menager-Tabourel E, Nader J, Baize N, Laly M, Lermite E, Bertrais S and Caroli-Bosc FX: The combination of everolimus and zoledronic acid increase the efficacy of gemcitabine in a mouse model of pancreatic adenocarcinoma. Oncotarget 9: 28069-28082, 2018.

15. Barrett-Lee P, Casbard A, Abraham J, Hood K, Coleman R, Simmonds P, Timmins H, Wheatley D, Grieve R, Griffiths G and Murray N: Oral ibandronic acid vs. intravenous zoledronic acid in treatment of bone metastases from breast cancer: A randomised, open label, non-inferiority phase 3 trial. Lancet Oncol 15: 114-122, 2014.

16. Coleman R, Cameron D, Dodwell D, Bell R, Wilson C, Rathbone E, Keane M, Gil M, Burkinshaw R, Grieve R, et al: Adjuvant zoledronic acid in patients with early breast cancer: Final efficacy analysis of the AZURE (BIG 01/04) randomised open-label phase 3 trial. Lancet Oncol 15: 997-1006, 2014.

17. Lee SH, Hong HS, Liu ZX, Kim RH, Kang MK, Park NH and Shin KH: TNFa enhances cancer stem cell-like phenotype via Notch-Hes1 activation in oral squamous cell carcinoma cells Biochem Biophys Res Commun 424: 58-64, 2012.

18. Shin KH, Bae SD, Hong HS, Kim RH, Kang MK and Park NH: miR-181a shows tumor suppressive effect against oral squamous cell carcinoma cells by downregulating K-ras. Biochem Biophys Res Commun 404: 896-902, 2011.

19. Yanamoto S, Kawasaki G, Yamada S, Yoshitomi I, Kawano T, Yonezawa H, Rokutanda S, Naruse T and Umeda M: Isolation and characterization of cancer stem-like side population cells in human oral cancer cells. Oral Oncol 47: 855-860, 2011.

20. Zhang P, Zhang Y, Mao L, Zhang Z and Chen W: Side population in oral squamous cell carcinoma possesses tumor stem cell phenotypes. Cancer Lett 277: 227-234, 2009.

21. Baba T,Naka K,Morishita S, Komatsu N,Hirao A and Mukaida N: MIP-1 $\alpha / C C L 3-$ mediated maintenance of leukemia-initiating cells in the initiation process of chronic myeloid leukemia. J Exp Med 210: 2661-2673, 2013.

22. Blanpain C,Buser R,PowerCA,Edgerton M,Buchanan C,Mack M, Simmons G, Clapham PR, Parmentier M and Proudfoot AE: A chimeric MIP-1 alpha/RANTES protein demonstrates the use of different regions of the RANTES protein to bind and activate its receptors. J Leukocyte Biol 69: 977-985, 2001

23. Schech AJ, Kazi AA, Gilani RA and Brodie AH: Zoledronic acid reverses the epithelial-mesenchymal transition and inhibits self-renewal of breast cancer cells through inactivation of NF- $\kappa B$. Mol Cancer Ther 12: 1356-1366, 2013.
24. Buhler H, Hoberg C, Fakhrian K and Adamietz IA: Zoledronic acid inhibits the motility of cancer stem-like cells from the human breast cancer cell line MDA-MB 231. In Vivo 30: 761-768, 2016.

25. Rouhrazi H, Turgan N and Oktem G: Zoledronic acid overcomes chemoresistance by sensitizing cancer stem cells to apoptosis. Biotech Histochem 93: 77-88, 2018.

26. Kijima T, Koga F, Fujii Y, Yoshida S, Tatokoro M and Kihara K: Zoledronic acid sensitizes renal cell carcinoma cells to radiation by downregulating STAT1. PLoS One 8: e64615, 2013.

27. Silva TA, Ribeiro FL, Oliveira-Neto HH, Watanabe S, Alencar Rde C, Fukada SY, Cunha FQ, Leles CR, Mendonça EF and Batista AC: Dual role of CCL3/CCR1 in oral squamous cell carcinoma: Implications in tumor metastasis and local host defense. Oncol Rep 18: 1107-1113, 2007.

28. da Silva JM, Moreira Dos Santos TP, Sobral LM, Queiroz-Junior CM, Rachid MA, Proudfoot AEI, Garlet GP, Batista AC, Teixeira MM, Leopoldino AM, et al: Relevance of CCL3/CCR5 axis in oral carcinogenesis. Oncotarget 8: 51024-51036, 2017.

29. Hsu CJ, Wu MH, Chen CY, Tsai CH, Hsu HC and Tang $\mathrm{CH}$ : AMP-activated protein kinase activation mediates CCL3-induced cell migration and matrix metalloproteinase- 2 expression in human chondrosarcoma. Cell Commun Signal 11: 68, 2013.

30. Liao YY, Tsai HC, Chou PY, Wang SW, Chen HT, Lin YM, Chiang IP, Chang TM, Hsu SK, Chou MC, et al: CCL3 promotes angiogenesis by dysregulation of miR-374b/ VEGF-A axis in human osteosarcoma cells. Oncotarget 7: 4310-4325, 2016.

31. Kim JH, Kim WS, Hong JY, Ryu KJ, Kim SJ and Park C: Epstein-Barr virus EBNA2 directs doxorubicin resistance of $\mathrm{B}$ cell lymphoma through CCL3 and CCL4-mediated activation of NF-kappaB and Btk. Oncotarget 8: 5361-5370, 2017.

32. Jin J, Colin P, Staropoli I, Lima-Fernandes E, Ferret C, Demir A, Rogée S, Hartley O, Randriamampita C, Scott MG, et al: Targeting spare CC chemokine receptor 5 (CCR5) as a principle to inhibit HIV-1 entry. J Biol Chem 289: 19042-19052, 2014.

33. Jiao X, Velasco-Velázquez MA, Wang M, Li Z, Rui H, Peck AR, Korkola JE, Chen X, Xu S, DuHadaway JB, et al: CCR5 governs DNA damage repair and breast cancer Stem cell expansion. Cancer Res 78: 1657-1671, 2018.

34. Mencarelli A, Graziosi L, Renga B, Cipriani S, D'Amore C, Francisci D, Bruno A, Baldelli F, Donini A and Fiorucci S: CCR5 antagonism by maraviroc reduces the potential for gastric cancer cell dissemination. Transl Oncol 6: 784-793, 2013.

35. Velasco-Velázquez M, Jiao X, De La Fuente M, Pestell TG, Ertel A, Lisanti MP and Pestell RG: CCR5 antagonist blocks metastasis of basal breast cancer cells. Cancer Res 72: 3839-3850, 2012.

36. Maehara O, Suda G, Natsuizaka M, Ohnishi S, Komatsu Y, Sato F, Nakai M, Sho T, Morikawa K, Ogawa K, et al: Fibroblast growth factor-2-mediated FGFR/Erk signaling supports maintenance of cancer stem-like cells in esophageal squamous cell carcinoma. Carcinogenesis 38: 1073-1083, 2017. 